\title{
Neoplasms of the Vagina following Cervical Carcinoma
}

\author{
Yew Cheong Choo, M.D., and David G. Anderson, M.D. \\ Division of Gynecologic Oncology, Department of Obstetrics and Gynecology at the University of \\ Michigan Hospital, Ann Arbor, Michigan 48109
}

Received July 24, 1981

\begin{abstract}
Neoplasm in the vagina which occurs following treatment of cervical carcinoma could represent a separate primary neoplasm, a "field" response to a common carcinogen, or be due to neoplastic transformation of tissue from previous radiation treatment. Fifty patients with a second neoplasm in the vagina were identified: 17 following total hysterectomy for carcinoma in situ of cervix; 4 following radical hysterectomy; and 29 following radiotherapy for invasive cervical carcinoma. Their clinical features, diagnosis, and treatment modalities are presented. Since vaginal neoplasm may occur long after treatment of the initial cervical carcinoma especially after previous radiotherapy, life-long follow-up of all patients with cervical carcinoma is mandatory.
\end{abstract}

\section{INTRODUCTION}

Primary neoplasms that arise in the vagina are infrequent; preinvasive carcinoma of the vagina was an entity seldom identified until recently [1], while invasive carcinoma which constitutes less than $2 \%$ incidence [2-4] is ranked as the second rarest malignancy of the female genital tract. Increasing attention, however, has now been focused on neoplasms that arise in the vagina following the apparent cure of a cervical carcinoma. Such neoplasms have often in the past been regarded as residual or recurrent carcinoma. They are now recognized as a distinctive entity that may occur in various forms: (1) Following a total hysterectomy for in situ carcinoma of the cervix, as carcinoma in situ $[5,6]$ or invasive carcinoma [7] of the vagina; (2) following radical hysterectomy for invasive carcinoma of the cervix, as carcinoma in situ or invasive carcinoma of the vagina [8]; and (3) following irradiation for invasive cervical carcinoma, as carcinoma in situ $[9,10]$, invasive carcinoma $[11,12]$, or sarcoma [13] of the vagina. This study is about a clinical analysis of a reasonably large number of such patients encountered in one institution and discusses their presentation, identification, and treatment.

\section{METHODS}

From the files of the Tumor Registry at the University of Michigan Hospital, 3239 patients were found to have cervical carcinoma between January 1957 and January 1980. The case records of all patients who had a subsequent neoplasm in the vagina during this 23 -year period were reviewed. Excluded from analysis 
were patients whose diagnosis of an independent second primary neoplasm in the vagina could not be ascertained and those in whom the initial cervical carcinoma had extended to the vagina on the hysterectomy specimen. Fifty patients with a second primary neoplasm in the vagina were thus identified, representing an incidence of $1.54 \%$ of patients who had cervical carcinoma. They all had a negative follow-up of at least a year, ranging from 1 to 39 years with a mean of 11.2 years. Other criteria employed in the identification of such cases include nonidentical histology between the two neoplasms and the presence of dysplasia in vaginal carcinoma in situ, or dysplasia and carcinoma in situ in invasive vaginal carcinoma suggesting their origin in the vagina rather than recurrent carcinoma.

\section{RESULTS}

Table 1 outlines the initial cervical carcinomas, their treatment, and the interval to the development of a second neoplasm in the vagina. It was noted that the mean age of patients who had in situ carcinoma of the cervix was 46.3 years and the mean interval to the development of carcinoma in situ and invasive carcinoma of the vagina was 5.8 and 12.6 years, respectively. On the other hand, the mean age of the patients who had invasive cervical carcinoma treated by irradiation was much younger (38.7 years), and their mean interval to the development of in situ and invasive neoplasms of the vagina was remarkably longer, at 9.9 and 22.7 years.

The presentation of the vaginal neoplasms was found to bear no relationship to the initial type of cervical carcinoma or its treatment. Noteworthy was the finding that 28 of 31 patients $(90.3 \%)$ with in situ and 8 of 19 patients $(42.1 \%)$

TABLE 1

Second Primary Neoplasms of the Vagina following Carcinoma of the Cervix

\begin{tabular}{|c|c|c|c|c|}
\hline & \multicolumn{4}{|c|}{ Interval to vaginal neoplasm (years) } \\
\hline & $1-5$ & $6-10$ & $>10$ & Total \\
\hline \multicolumn{5}{|l|}{$\begin{array}{l}\text { Prior total hysterectomy for CIS } \\
\text { of cervix }\end{array}$} \\
\hline CIS of vagina & 5 & 5 & 2 & 12 \\
\hline Invasive carcinoma of vagina & 1 & 0 & 4 & 5 \\
\hline Total & & & & 17 \\
\hline \multicolumn{5}{|l|}{$\begin{array}{l}\text { Prior radical hysterectomy for in- } \\
\text { vasive carcinoma of cervix }\end{array}$} \\
\hline CIS of vagina & 1 & 2 & 1 & 4 \\
\hline Total & & & & 4 \\
\hline \multicolumn{5}{|l|}{$\begin{array}{l}\text { Prior radiation for invasive carci- } \\
\text { noma of cervix }\end{array}$} \\
\hline CIS of vagina & 2 & 7 & 6 & 15 \\
\hline Invasive carcinoma of vagina & 0 & 3 & 9 & 12 \\
\hline Sarcoma of vagina & 0 & 0 & 2 & 2 \\
\hline Total & & & & 29 \\
\hline
\end{tabular}


TABLE 2

Location of Vaginal Neoplasms

\begin{tabular}{lcc}
\hline & $\begin{array}{c}\text { CIS of } \\
\text { vagina }\end{array}$ & $\begin{array}{c}\text { Invasive } \\
\text { neoplasm } \\
\text { of vagina }\end{array}$ \\
\hline Apex & 29 & 12 \\
Upper lateral & 2 & 5 \\
Midposterior & - & 1 \\
Distal anterior & - & 1 \\
Total & 31 & 19 \\
\hline
\end{tabular}

with invasive vaginal neoplasm were asymptomatic. A red granular lesion was described in 15 of 31 patients (43.4\%) with carcinoma in situ and 6 of 19 patients (31.6\%) with invasive carcinoma of the vagina. Fourteen patients with in situ carcinoma were identified on the basis of an abnormal Pap smear alone.

The locations of the vaginal neoplasms are as shown in Table 2. The most common site was the apex, followed by the lateral aspects of the upper vagina. One patient had the neoplasm located in the mid- and another, the distal vagina.

Neoplasms in the cervix and vagina of dissimilar histology were found in three patients, all following irradiation. In one patient, the initial cervical carcinoma was adenocarcinoma while the vaginal carcinoma was squamous. In the other two patients, the cervical carcinoma was squamous while the vaginal neoplasm was sarcoma-one was carcinosarcoma and the other, fibrosarcoma; both occurred 13 years after irradiation for cervical carcinoma.

Significantly, most of the invasive carcinomas that arose in the vagina were Stage I lesions. Of the 18 patients with invasive carcinoma of the vagina following carcinoma in situ or invasive carcinoma of the cervix, only $8(27.8 \%)$ had extension beyond the vagina, and in 2 patients the neoplasm had infiltrated the pelvic side wall.

The treatment modalities for the 31 patients with carcinoma in situ of the vagina are as shown in Table 3 . The most common treatment was vaginectomy

TABLE 3

Treatment and Status of Patients with CiS of Vagina foitowing Cervical Carcinoma

\begin{tabular}{lcl}
\hline & No. & Status \\
\hline $\begin{array}{l}\text { Vaginectomy } \\
\text { NAHBSO and vaginectomy }\end{array}$ & 24 & $\begin{array}{c}\text { NED } 22 \text { (8 months-18 years); I died of recurrent } \\
\text { invasive Ca of vagina; 1 died of intercurrent } \\
\text { disease }\end{array}$ \\
$\begin{array}{l}\text { NED (1-6 years) } \\
\text { Vaginal radium }\end{array}$ & 4 & NED (6 years) \\
\end{tabular}

Note. TAHBSO, total abdominal hysterectomy and bilateral salpingo-oophorectomy; RH,PLND, radical hysterectomy and pelvic lymphadenectomy. 
which was performed in 24 patients-20 partial and 4 total, and 5 patients had simultaneous vaginoplasty with split thickness skin graft as well. The availability of additional surgical tissue led to an unexpected diagnosis of superficial invasion in 4 of these patients. A total hysterectomy, bilateral salpingo-oophorectomy, and upper vaginectomy were performed on 4 patients who previously had irradiation for an invasive cervical carcinoma. One patient underwent radical hysterectomy, pelvic lymphadenectomy, and total vaginectomy when the original biopsy showed questionable "microinvasion." Two patients were treated with vaginal radium. One follow-up examination, 1 patient was noted to have recurrent carcinoma in situ of the vagina and she was treated with further excision. Two patients died -1 from unrelated disease and the other from progressive disease 2 years following partial vaginectomy for incidental superficial invasive disease.

Table 4 shows the treatment for patients with invasive neoplasms of the vagina. Irradiation was carried out on all five patients with invasive vaginal carcinoma following total hysterectomy for in situ carcinoma of the cervix; only two are alive at 3 and 6 years. Radiation was also given to five other patients who previously had irradiation for invasive carcinoma of the cervix; three died within a year, while the fourth sustained numerous episodes of bowel fistulae requiring multiple corrective surgery and died without disease 3 years later. The lone survivor is disease free for 8 months. Five patients underwent total pelvic exenteration; one died of postoperative sepsis, the rest are alive from 6 months to 7 years. Three patients with superficial invasive disease underwent radical vaginectomy; one died of progressive disease within a year and one is alive for 5 years. The third patient is of interest in that she developed a superficially invasive carcinoma on the vaginectomy skin graft 7 years later and was further treated by an exenteration.

That such patients may be prone to other neoplasms was demonstrated in four patients. Two patients had vulvar carcinoma in situ that occurred synchronously with vaginal carcinoma in situ. One patient, in a 12-year interim period between an invasive cervical carcinoma and an in situ vaginal carcinoma, developed a breast carcinoma and a meningioma, both successfully treated. The fourth patient had five independent neoplasms over a span of 17 years. In sequence, they are carcinoma in situ of the cervix, carcinoma in situ of the vagina, hemangioblastoma

TABLE 4

Treatment and Status of Patients with Invasive Primary Neoplasm of the Vagina following Cervical Carcinoma

\begin{tabular}{|c|c|c|}
\hline & No. & Status \\
\hline Radical vaginectomy & 3 & $\begin{array}{l}1 \text { died of progressive disease; } 1 \text { recurrent CIS treated by } \\
\text { local excision; } 1 \text { recurrence treated by exenteration }\end{array}$ \\
\hline Total peivic exenteration & 5 & NED 4 (6 months-6 years); 1 died of postoperative sepsis \\
\hline Radiation $^{a}$ & 10 & NED 4 ( 6 months -6 years); 6 died of disease \\
\hline Chemotherapy & 1 & Died of disease \\
\hline
\end{tabular}

${ }^{a}$ Five patients had prior irradiation. 
of the cerebellum, carcinoma of the breast, and invasive carcinoma of the vulva; she died of her last acquired neoplasm.

\section{DISCUSSION}

When carcinoma of the vagina occurs following carcinoma of the cervix, the question frequently raised is whether it is a residual or recurrent carcinoma, or represents a new primary lesion. Their distinction, though not always clear cut, is nevertheless possible.

In situ carcinoma of the vagina is rare without a preceding or synchronous carcinoma in the cervix $[5,6,9]$. The reported incidence of "recurrent" carcinoma in situ following hysterectomy for carcinoma in situ of the cervix ranged from 0.7 to $6.6 \%$ [14-16]. A preoperative mapping of the lesion in a patient with carcinoma of the cervix should be a prerequisite in order to exclude extension of the lesion on to the vagina or the presence of multicentric carcinoma of the cervix and vagina. When the initial cervical lesion is completely cxtirpated as determined by pathological examination of the specimen and following a period of negative follow-up examinations, any lesion that appears in the vagina must be considered a new primary lesion.

Invasive vaginal carcinoma that arises long after a total hysterectomy for in situ carcinoma of the cervix has been considered a curious entity $[7,16,17]$. Coppleson [17] thought that some of these patients originally might have an invasive cervical carcinoma that extended into the vagina. The late occurrence of this cancer after a mean interval of $\mathbf{1 2 . 6}$ years in our five patients suggests that it is an independent neoplasm.

Carcinoma in situ and primary invasive vaginal carcinoma following radical hysterectomy for invasive carcinoma of the cervix have rarely been reported. Kanbour [8] documented five such patients with in situ and four with invasive carcinoma. We identified only four patients with in situ carcinoma and two among them received adjunctive postoperative irradiation as well. No patients were found to have a primary invasive vaginal carcinoma when recurrent invasive cervical carcinoma was excluded. It is generally accepted that 5-year survival following treatment of invasive cervical carcinoma is an accurate measure of curability [18]. Therefore, an invasive vaginal carcinoma that arises more than 5 years after radical hysterectomy for invasive cervical carcinoma should lead to a strong suspicion of a possible new lesion. It is likely that the removal of the upper half of the vagina may also remove the site at risk for carcinoma in the vagina, considering the fact that the cervix and the upper vagina are of Mullerian origin and they provide a common embryological field that can be acted upon by a common carcinogen.

Neoplasms arising in the vagina following irradiation may suggest radiation carcinogenicity. This is evidenced by such neoplasms occurring long after irradiation, many of them possessing histological features suggestive of de novo origin when concomitant dysplasia and carcinoma in situ are also found [19-21]. The implication of radiation carcinogenicity is especially strong in our patients. Twenty-seven of twenty-nine patients (93.1\%) developed a neoplasm in the vagina 
more than 5 years, and 17 of them (58.6\%) more than 10 years after irradiation (Table 1). Noted also were 2 patients who developed sarcoma of the vagina following radiotherapy. Sarcoma arising in the irradiated soft tissue and bone have been well documented in the literature [22,23]. Furthermore, 8 of 15 patients (53.3\%) with carcinoma in situ of the vagina had associated dysplasia, and 7 of 12 patients $(58.3 \%)$ with invasive squamous carcinoma of the vagina demonstrated dysplasia and carcinoma in situ as well.

The observation in this study that young patients irradiated for invasive cervical carcinoma are at risk of developing a second neoplasm in the vagina warrants attention. Eight of twenty-nine patients were below the age of 35 when they received radiotherapy for their cervical cancer. Such an observation was first reported by Barrie and Brunschwig [24] in 1970. They found 9 of 11 patients in their $20 \mathrm{~s}$ or $30 \mathrm{~s}$, when irradiated for their cervical carcinoma, subsequently developed a second cancer in the cervix 15 to 39 years later. Futoran and Nolan [13] also reported a series of 76 patients 38 years of age or younger, 12 of whom subsequently developed carcinoma in the vagina following irradiation for their cervical cancer. These young patients live long enough to develop a second neoplasm in the irradiated vagina, thus life-long surveillance in this unique group of patients is mandatory.

While invasive neoplasm in the vagina is frequently evident on examination, that of in situ carcinoma often is obscure. The presence of a "pink blush," as described by Young [25] to be characteristic of carcinoma in situ of the vagina, should arouse suspicion. Vaginal cytology is undoubtedly useful; misinterpretation can, however, occur in the presence of extreme atrophy or radiation atypia. Colposcopic examination of the vagina may also at times be nonproductive as the lesion may not be delineated owing to radiation atrophy. In such a situation, a 2-week course of topical or oral estrogen will often help to outline the lesion. Particular attention should be directed to the angles of the apex of vagina as they are often the hidden sites of carcinoma and such areas are difficult to examine and biopsy.

The treatment of vaginal neoplasms should be individualized, taking into consideration the patient's age, type of malignancy, extent of disease, previous therapy, and the need to preserve sexual function. Although some may find topical application of $5 \mathrm{FU}$ more acceptable in carcinoma in situ of the vagina [26], our preference is vaginectomy. A limited partial vaginectomy or an excision can be performed following colposcopic mapping. Vaginal obliteration or stenosis can be avoided by a vaginoplasty or the use of vaginal dilator alone. Its important advantage lies in the availability of additional tissue for diagnosis lest a superficial or frank invasive carcinoma should be missed. Vaginal radium is also an equally effective method for treating carcinoma in situ of the vagina especially if the carcinoma is multicentric. Other conservative methods such as immunotherapy with DNCB [27] and laser treatment [28] are still experimental. In patients who have invasive neoplasm of the vagina, the choice is one of radical surgery or exenteration in patients who have previously been irradiated, or primary irradiation in patients who previously had surgery for the cervical carcinoma. Reir- 
radiation for cure, no matter how long the interim period may be, is ineffective and fraught with potential complications and is not recommended.

\section{REFERENCES}

1. Gallup, D. G., and Morley, G. W. Carcinoma in situ of the vagina: A study and a review, Obstet. Gynecol. 45, 334-340 (1975).

2. Merrill, J. A., and Bender, W. T. Primary carcinoma of the vagina, Obstet. Gynecol. 11, 3-11 (1958).

3. Frick, H. C., II, Jaco, H. W., and Taylor, H. C., Jr. Primary carcinoma of the vagina, Amer. J. Obstet. Gynecol. 101, 695-703 (1969).

4. Dunn, C. J., and Napier, J. G. Primary carcinoma of the vagina, Amer. J. Obstet. Gynecol. 96, 1112-1116 (1966).

5. Ferguson, J. H., and Maclure, J. G. Intraepithelial carcinoma, dysplasia, and exofoliation of cancer cells in the vagina mucosa, Amer. J. Obstet. Gynecol. 87, 326-336 (1967).

6. Hummer, W. K., Mussey, E., Decker, D. G., and Doclerty, M. B. Carcinoma in situ of vagina, Amer. J. Obstet. Gynecol. 108, 1109-1116 (1970).

7. Cramer, J. K. Invasive squamous cell carcinoma of the vagina following surgery for carcinoma in situ of cervix, Med. Ann. D.C. 34, 115-117 (1965).

8. Kanbour, A. I., Klionsky, B., and Murphy, A. I. Carcinoma of the vagina following cervical cancer, Cancer 34, 1838-1841 (1974).

9. Rutledge, F. Cancer of vagina, Amer. J. Obstet. Gynecol. 97, 635-665 (1967).

10. Koss, C. G., Melamed, M. R., and Daniel, W. W. In situ epidermoid carcinoma of cervix and vagina following radiotherapy for cervical cancer, Cancer 14, 353-360 (1961).

11. Geisler, H. S. Carcinoma of the cervix or vagina 10 or more years after therapy: Recurrence or new primary, J. Indiana Med. Assoc, 67, 711-712 (1974).

12. Pride, G. L., and Buchler, D. A. Carcinoma of vagina $\mathbf{1 0}$ or more years following pelvic irradiation therapy, Amer. J. Obstet. Gynecol. 127, 513-517 (1977).

13. Futoran, R. J., and Nolan, J. Stage I carcinoma of the uterine cervix in patients under 40 of age, Amer. J. Obstet. Gynecol. 125, 790-797 (1976).

14. Coppleson, M., and Rcid, B. Preclinical carcinoma of the cervix uteri: Its nature, origin, and management, Pergamon, Oxford, p. 191 (1967).

15. Funnell, J. D., and Merrill, J. A. Recurrence after treatment of carcinoma in situ of cervix, Surg. Gynecol. Obstet. 117, 15-19 (1963).

16. Kolstad, P., and Klem, V. Long term follow-up of 1121 cases of carcinoma in situ, Obstet. Gynecol. 48, 125-129 (1976).

17. Coppleson, M. Management of preclinical cancer of the cervix, in The cervix (Jordan and Singer, Eds.), Saunders, London/Philadelphia/Toronto, p. $455^{\prime}$ (1976).

18. DiSaia, P. J., Morrow, C. P., and Townsend, D. E. Synopsis of gynecological oncology, Wiley, New York, p. 75 (1974).

19. Geelhoed, G. W., Henson, D. E., Taylor, P. T., and Ketcham, A. S. Carcinoma in situ of the vagina following treatment for carcinoma of cervix: A distinct clinical entity, Amer. J. Obstet. Gynecol. 124, 510-516 (1976).

20. Murrad, T. M., Durant, J. R., Maddox, W. Z., and Dowling, E. A. The pathological behavior of primary vaginal carcinoma and its relationship to cervical cancer, Cancer 35, 787-794 (1975).

21. Durrance, F. Y., Fletcher, G. H., and Rutledge, F. N. Analysis of central recurrent disease in Stages I and II squamous cell carcinoma of the cervix on intact uterus, Amer. J. Roentgenol. Ther. Nucl. Med. 106, 831-838 (1969).

22. Kim, J. H., Chu, F. C., Woodward, H. Q., Huvos, A., and Cantin, J. Radiation-induced soft tissue and bone sarcoma, Radiology 129, 501-508 (1978).

23. Czesnin, K., and Wronkowski, Z. Second malignancies of the irradiated area in patients treated for uterine cervix cancer, Gynecol. Oncol. 6, 309-315 (1979).

24. Barrie, J. R., and Brunschwig, A. Late second cancers of the cervix after apparent successful initial radiation therapy, Amer. J. Roentgenol. Ther. Nucl. Med. 108, 109-112 (1970). 
25. Young, P. A. Clinical findings in early lesions of the cervix, In dysplasia, carcinoma in situ and microinvasive carcinoma of the cervix uteri (L. A. Gray, Ed), Thomas, Springfield, Ill., p. 182 (1964).

26. Ballon, S. C., Roberts, J. A., and Lagasse, L. D. Topical S-fluorouracil in the treatment of intraepithelial neoplasia of the vagina, Obstet. Gynecol. 54, 163-166 (1979).

27. Guthrie, D., and Way, S. 5 FU or DNCB for non-clinical vaginal cancer, Lancet 1, 906 (1976).

28. Stafl, A., Wilkinson, E. J., and Mattingly, R. Laser treatment of cervical and vaginal neoplasia, Amer. J. Obstet Gynecol. 128, 128-136 (1977). 